\title{
BMJ Open Evaluation of a hospital-based integrated model of eye care for diabetic retinopathy assessment: a multimethod study
}

\author{
Janet C Long (10 , ${ }^{1}$ Brette Blakely, ${ }^{1}$ Zeyad Mahmoud (1) , ${ }^{1}$ Angelica Ly, ${ }^{2,3}$ \\ Barbara Zangerl, ${ }^{2,3}$ Michael Kalloniatis, ${ }^{2,3}$ Nagi Assaad, ${ }^{4}$ Michael Yapp, ${ }^{2,3}$ \\ Robyn Clay-Williams (D) , Jeffrey Braithwaite (D) ${ }^{1}$
}

To cite: Long JC, Blakely B, Mahmoud Z, et al. Evaluation of a hospital-based integrated model of eye care for diabetic retinopathy assessment: a multimethod study. BMJ Open 2020;10:e034699. doi:10.1136/ bmjopen-2019-034699

- Prepublication history and additional material for this paper are available online. To view these files, please visit the journal online (http://dx.doi. org/10.1136/bmjopen-2019034699).

Received 03 0ctober 2019 Revised 14 February 2020 Accepted 03 April 2020

Check for updates

(C) Author(s) (or their employer(s)) 2020. Re-use permitted under CC BY-NC. No commercial re-use. See rights and permissions. Published by BMJ.

\section{${ }^{1}$ Australian Institute of} Health Innovation, Macquarie University, Sydney, New South Wales, Australia

${ }^{2}$ School of Optometry and Vision Health, University of New South Wales, Sydney, New South Wales, Australia

${ }^{3}$ Centre for Eye Health, Sydney, New South Wales, Australia

${ }^{4}$ Prince of Wales Clinical School, University of New South Wales, Sydney, New South Wales, Australia

Correspondence to

Dr Janet C Long;

janet.long@mq.edu.au

\section{ABSTRACT}

Objectives Diabetic eye disease is a leading cause of blindness but can be mitigated by regular eye assessment. A framework of issues, developed from the literature of barriers to eye assessment, was used to structure an examination of perceptions of a new model of care for diabetic retinopathy from the perspective of staff using the model, and health professionals referring patients to the new service.

Design Multimethod: interviews and focus groups, and a separate survey.

Setting A new clinic based on an integrated model of care was established at a hospital in outer metropolitan Sydney, Australia in 2017. Funded jointly by Centre for Eye Health (CFEH) and the hospital, the clinic was equipped and staffed by optometrists who work alongside the ophthalmologists in the existing hospital eye clinic. Participants Five (of seven) hospital staff working in the clinic (ophthalmologists and administrative officers) or referring to it from other departments (endocrinologists); nine optometrists from CFEH who developed or worked in the clinic; 10 community-based optometrists as potential referrers.

Results The new clinic was considered to have addressed known barriers to eye assessment, including access, assistance for patients unable/unwilling to organise eye checks and efficient management of human resources. The clinic optimised known drivers of this model of care: providing clear scope of practice and protocols for shared care between optometrists and ophthalmologists, good communication between referrers and eye professionals and a collegial approach promoting interprofessional trust. Remaining areas of concern were few referrals from general practitioners, fewer referrals from hospital endocrinologists than expected and issues with stretched administrative capacity. There were also perceived mismatches between the priorities of hospital management and aims of the clinic.

Conclusions The new model was considered to have addressed many of the barriers to assessment. While there remain issues with the model, there were also unexpected benefits.

\section{INTRODUCTION}

Diabetes is a chronic metabolic condition characterised by an inability to produce or
Strengths and limitations of this study

- Integrated care models are known to overcome some barriers but can give rise to others making early evaluation important.

- A comprehensive new framework was developed from the literature on barriers to accessing eye assessments to aid analysis of this new model of integrated care.

- All staff associated with the hospital-based clinic were invited to participate; all but two took part (88\%).

- Our inability to collect data from the general practitioners in the area means we have no first-hand information about the acceptability of the clinic from their perspective.

- The hospital-based clinic has many unique contextual features that make some of the evaluation findings not generalisable.

effectively use insulin. Treatment requires lifelong multidisciplinary management aimed at maintaining healthy blood glucose levels to prevent complications. High blood glucose levels can damage microvasculature throughout the body, including the eyes, where damage can lead to irreversible vision loss.

Based on a survey conducted by the Australian Bureau of Statistics, $5.7 \%$ of Australians over the age of $18 \mathrm{had}$ diabetes in $2017-2018^{1}$ (around 1.2 million people). The prevalence of the disease has increased from $3.3 \%$ since 2011. It has increased for both males and females, and continues to be more common among males (by $5.5 \%$ ). ${ }^{1}$

As is the case with most chronic diseases, older populations show increased rates of diabetes. In 2017-2018, 15.45\% of adults aged between 65 and 74 and almost one in five adults over the age of 75 years $(18.6 \%)$ were diagnosed with diabetes. ${ }^{1}$ This has significant ramifications for health service delivery as the population ages. In 2017, 15\% of Australians 
were aged 65 and over, ${ }^{2}$ but this number is projected to more than double by $2055 .{ }^{3}$ The complexities associated with the management of diabetes requires the addition of sustainable long term resources, often leading to a substantial increase in costs. ${ }^{4}$ Chronic conditions also add economic and social burdens on patients which raise questions related to adherence to treatment and optimal disease management. ${ }^{5}$

In Australia, diabetic retinopathy is among the top five conditions leading to vision loss. ${ }^{6}$ Almost all patients with type 1 and a significant majority of patients with type 2 diabetes will develop diabetic eye disease within 20 years of their diagnosis. For people with type 2 diabetes, whose diagnosis may be significantly delayed, eye disease may already be present at first presentation to a health professional. A decrease over the last decade in numbers of people with vision loss due to diabetic retinopathy in the UK has been attributed to improved, routine assessment of people with diabetes combined with improved glycaemic control. ${ }^{7}$

General practitioners (GPs) coordinate the overall care for patients with diabetes. National Health and Medical Research Council guidelines around eye care of people with diabetes specifies an eye examination by an optometrist or ophthalmologist at least every 2 years depending on other risk factors. ${ }^{8}$ In Australia, diabetic retinopathy assessment may be carried out in a number of different settings. Both community-based optometry practices and hospital-based ophthalmology clinics provide comprehensive assessment typically provided at no charge to the patient. In private ophthalmology practices, patients may incur a significant extra out-of-pocket expense above what is covered by the national government funded healthcare scheme (Medicare). Additional diagnostic testing commonly performed by ophthalmologists and optometrists as part of diabetic retinopathy assessment (eg, fundus photography, optical coherence tomography) typically do not attract Medicare rebates, often resulting in out-of-pocket expense for patients. Significant waitlists exist in some areas for both hospital and private ophthalmology consults. ${ }^{9} 10$

The Centre for Eye Health (CFEH) is an initiative of the University of New South Wales (NSW) and Guide Dogs NSW/ACT. CFEH provides advanced diagnostic and eye disease management services at no cost to clients with the aim of intervening early to prevent vision loss. ${ }^{11}$ The primary clinic is based on the inner metropolitan University campus. In 2017, CFEH introduced a collaborative interprofessional model of care in conjunction with a public, outer metropolitan hospital in Sydney, aimed at monitoring and treating patients with diabetic retinopathy. The selected model of care was an integrated service located within the hospital, with the CFEH clinic equipped with specialist diagnostic equipment and staffed by optometrists who work alongside the hospital Eye clinic staff-ophthalmology registrars and consultants. We use Kodner and Spreeuwenberg's ${ }^{12}$ definition of integrated care: Integration is an approach designed to
Box 1 Issues related to the model of care used for assessment and management of people with diabetes. These issues formed the framework for our analysis

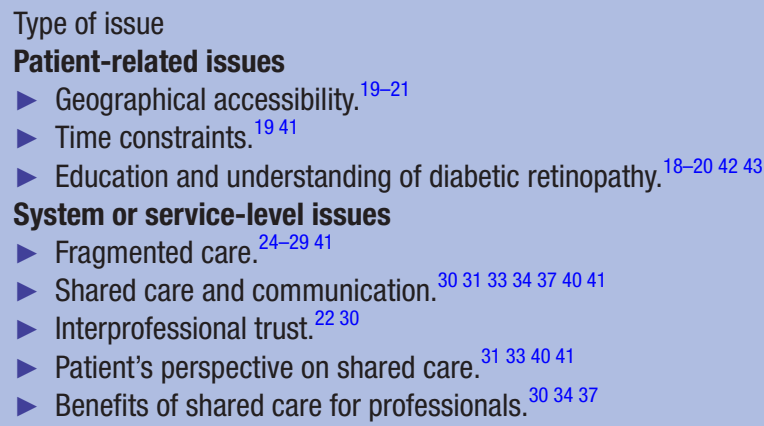

create connectivity, alignment and collaboration within and between the cure and care sectors. Here in the CFEH model of care, ongoing assessment and treatment for people with diabetes is organised to be managed across different health professionals, sharing patient information and deliberately linking eye services with primary health and endocrinology.

This study aimed to explore health professionals' (working within the clinic) and referrers' (into the clinic) perceptions of the acceptability, appropriateness and feasibility of the new model of eye care, and identify risks, drivers and barriers to optimal clinic functioning. The study forms part of a larger evaluation project of the clinic and its integrated model of care.

\section{Development of the evaluation framework}

Our examination of the CFEH model of care was structured using a framework developed from an analysis of relevant international literature. Box 1 shows the framework and the issues considered. Issues identified around the model of care used in the management of patients with diabetic retinopathy fall broadly into two categories: issues relating to the patient and issues relating to the system or service level.

\section{Patient-related issues}

Successful treatment of diabetic retinopathy is greatly enhanced by early detection and timely referrals. ${ }^{13-15}$ Adherence to the recommended guidelines for eye examinations, however, is reported to be as low as $50 \%$ for non-Indigenous Australians, ${ }^{16}$ with a National Eye Health Survey reporting that $26.2 \%$ of Indigenous Australians with diabetes reported that they had never undergone a diabetic eye examination, compared with $15.3 \%$ of nonIndigenous participants. ${ }^{17}$

The major reason for non-adherence by both Indigenous and non-Indigenous Australians to the recommended eye examination schedule was lack of awareness of the need. ${ }^{17}$ This pattern is consistent with other developed countries where patients' education, understanding of and adherence to assessment for diabetic retinopathy were also considered to have an impact on attendance 
levels. Both patients and providers interviewed for a study at two sites in England pointed to the lack of awareness about diabetic retinopathy as a significant barrier to assessment attendance. ${ }^{18}$ Hartnett $e t$ al showed that although patients considered they received adequate education about their disease, there was much confusion regarding symptoms and risks of diabetic retinopathy. ${ }^{19}$

Multiple studies have also identified accessibility issues as a significant barrier to assessment attendance of patients with diabetic retinopathy. Travel distance, assessment location as well as the availability and quality of public transport were all associated with attendance rates. ${ }^{190}$ A study examining assessment rates in the USA revealed that patients were less likely to attend if they lived more than eight miles away from the assessment facility. ${ }^{21}$ Long-waiting times coupled with scheduling issues, lengthy consultations and waiting times on the day of assessment, all contribute to lower attendance rates. ${ }^{19}$

Solutions to improve patient's uptake of diabetic retinopathy assessment have also been identified in the literature. The integration of diabetic retinopathy assessment with other diabetes-related medical check-ups is a way to reduce the inconvenience of the assessment process and the burden diabetes has on patients. ${ }^{2022} 23$

\section{System-level issues}

Patients with diabetes commonly have several specialist and generalist healthcare providers (eg, GPs, optometrists or ophthalmologists, dieticians, endocrinologists) which increases the risk of miscommunication. Referral and communication issues have been identified as barriers to assessment attendance. The absence of an assessment recommendation by a GP was shown in some cases to have a negative effect on attendance of patients. ${ }^{24-28}$ At a systems level, studies have identified the lack of communication between health professionals as a contributory factor of low assessment attendance. ${ }^{29}$ The lack of communication was in some cases attributed to inflexible and incompatible information technology systems and in others to the absence of communication channels and protocols that enable effective teamwork. ${ }^{22}$

Beyond improved communication, there is evidence of the effectiveness of shared care models. Shared care models contribute to closing the widening gap between supply and demand of hospital-based assessment provided by ophthalmologists. ${ }^{30}{ }^{31}$ Involving optometrists and other healthcare professionals in the assessment of patients with diabetic retinopathy contributes to a more efficient utilisation of the workforce, especially given the shortage in the number of ophthalmologists. ${ }^{30}$ Shared models of care have been shown to give patients quicker access to assessment appointments and allowed routine follow-up sessions to be scheduled more conveniently. ${ }^{22}$ By working together, hospital-based ophthalmologists and community-based optometrists were shown to affect waitlist issues by providing patients with localised, more accessible care options. ${ }^{22}$
The existence of shared care models in other areas of eye care services demonstrates practitioners' openness to such arrangements. ${ }^{30-32}$ A recent study examining the attitudes and perceptions of eye care workers regarding shared management of people with diabetic retinopathy in Pakistan revealed that $100 \%$ of participating medical practitioners believed it was appropriate for optometrists to be involved in assessment while $61 \%$ believed that orthoptists were appropriate. ${ }^{33}$ The authors of the Pakistani study concluded that tasks such as education, assessment and monitoring all have the potential to be performed by eye care professionals who have adequate training but are not necessarily ophthalmologists (ie, optometrists). ${ }^{30}$ Task sharing as an alternative to current care delivery models was found to have made assessment and monitoring more accessible to participants especially in the context of an increasing number of patients. ${ }^{30}$

Issues around interprofessional trust between stakeholders was identified as a barrier to the implementation of such models. In a shared care arrangement, ophthalmologists must trust the optometrists' ability to deliver high quality care despite having few or no prior working relationships with them. ${ }^{22}$ Standards and guidelines are suggested ways that interprofessional trust and partnerships could be built. ${ }^{34}$

In Australia, shared care models for the management of chronic eye conditions such as glaucoma are encouraged by the National Health and Medical Research Council, ${ }^{35}$ and the Optometry Board of Australia. ${ }^{36}$ Under such a model, the ophthalmologist and optometrist develop a written management plan that covers the treatment goal, roles and responsibilities of the two practitioners and create an appropriate review schedule. Information related to the patient and the progress of their care management plan are sent to the patient's GP to promote an integrated approach. ${ }^{31}{ }^{37}$ GPs are key players in the development of such arrangements as they are often the initiator of referrals. ${ }^{22} 31$

From a patient perspective, there is mixed evidence of the acceptability of optometrists rather than ophthalmologists conducting their eye assessment. ${ }^{38} 39$ Some patients in a shared-care glaucoma clinic highlighted the convenience of attending an assessment by an optometrist over having to attend a hospital based assessment. ${ }^{31}$ This was because optometrists based in the community do not require a GP referral, are often located in shopping districts with adequate parking and appointments are easy to make at short notice. In terms of quality, task sharing between ophthalmologists and optometrists was not believed to have any negative impact on the quality of care delivered to patients in one study. ${ }^{33}$ Banes et al found evidence that optometrists with adequate training who work in supportive work environments can make sound clinical decisions and provide high-quality care to patients with glaucoma. ${ }^{40}$

Shared care models are not only beneficial for patients; they also yield benefits for healthcare professionals. There is evidence that both ophthalmologists and optometrists 
value the professional growth opportunities enabled by such models. ${ }^{31}$

Using this framework of known barriers and drivers, we examined perceptions of the acceptability, appropriateness and feasibility of the new model of eye care from the perspective two broad groups. The first group were hospital based: the eye health professionals (ophthalmologists, optometrists) and administrative staff working in the hospital clinic and hospital-based endocrinologists consulting with and referring to the clinic. The second group were community based: health professionals, (GPs and community-based optometrists) referring into the clinic (called hereafter 'referrers'). We also identified risks, unintended consequences and facilitators for optimal clinic functioning.

\section{METHODS}

The study applied a multimethod design, using interviews and focus groups involving key stakeholders and a separate telephone survey of potential external referrers. Participants for the interviews and focus groups were staff members of the hospital, drawn from those working in the ophthalmology clinic and those referring to the clinic from other departments of the hospital. CFEH optometrists who staffed and managed the clinic were also participants. Staff members of the hospital were approached initially by the CFEH's clinic directors with an invitation to take part. Staff who were happy to participate contacted the researchers who after providing information on the study, negotiated a convenient, private place and time for interviews.

The attitudes and experiences of the extended referral network of local community-based optometrists (29 optometry practices) were canvassed using a structured survey delivered over the phone. The surveys were administered by CFEH staff. These practices had all received advertising and referral information about the new clinic from CFEH previously. Analysis was undertaken by researchers and synthesised where possible with project data.

Interviewees and focus group participants were given a participant information sheet and asked to sign the consent form. Opportunity for questions and any concerns was given along with the provision of the research team's contact details. All participants were advised that they could withdraw consent at any time without fear of consequence.

\section{Data collection}

Two focus groups were used to capture information from the CFEH clinic staff to ascertain expectations, experiences and patient pathway data. Focus groups were semi-structured and audio recorded for transcription. Focus groups (JCL and BB) and interviews (JCL) were conducted by $\mathrm{PhD}$ qualified social science researchers with experience in bioethics (BB) and health systems (JCL). Researcher JCL disclosed her previous clinical role as a senior ophthalmic nurse and that facilitators were 'neutral' coming from an external university. The focus group schedule is in online supplementary appendix 1 .

Referring endocrinologists, ophthalmologists and administrative officers within the hospital $(n=7)$ were invited to be interviewed in order to gain a more detailed analysis of their experiences of the new clinic. Interviews were conducted face to face at a private venue convenient for participants. Two participants asked not to be recorded so notes were taken instead. Audio recordings of the interviews were transcribed, and two independent researchers used NVivo V.12 software for analysis (JCL and ZM). Transcripts were not returned to the participants for comment. The interview schedule is in online supplementary appendix 2 . The phone survey questions are in online supplementary appendix 3 .

\section{Analysis}

NVivo V.12 software was used to organise and analyse interview and focus group data. Since the model of shared care was novel, data from interviews and focus groups were initially used to develop an understanding of processes of patient care within the new clinic model. This was to ensure our analysis did not suffer from any pre-suppositions or assumptions regarding processes. We then deductively coded the data using our framework of known barriers and facilitators from the literature on shared care models of assessment for diabetic retinopathy. We also used an inductive approach to identify any other issues discussed that were outside the framework. Coding was done independently by two researchers (ZM and JCL), and then compared and refined. Results were discussed and validated by the full research team.

The phone survey data were collated and nonidentifiable data downloaded into SPSS V.24 for analysis. Scores for Likert scale questions that were stated negatively were reversed for analysis. Means, median and mode of each question were calculated. The information was triangulated with other data wherever appropriate.

\section{Patient and public involvement}

No patients were involved in this study as patient satisfaction and outcomes were collected by a different project team. Only healthcare professionals and administrative staff were involved in this study.

\section{RESULTS \\ Participants}

Interviews and focus groups were carried out between September and November 2018. All but two of the seven invited staff members employed by the hospital that were associated with the CFEH clinic took part in interviews. As we undertook to ensure our small number of participants would not be identifiable, we only characterise the group as including endocrinologists, ophthalmologists and administrative officers. Staff not participating did not reply to the invitation. Participants had varying levels of 
Table 1 Summary of findings using the framework derived from the literature on barriers to assessment of diabetic retinopathy

\begin{tabular}{ll}
\hline Identified barriers & Findings \\
\hline Patient-related issues & \\
Geographical accessibility & Distance and ease of access mentioned by all \\
& Location well serviced by public transport \\
& Pefents appreciated ease of access \\
& Staff appreciated that clinic complements other services offered at the hospital benefitting \\
& inpatients and outpatients
\end{tabular}

System or service-level issues

Fragmented care and integration of services

\begin{tabular}{ll} 
Shared care and & Comprehensive report returned to referrers was valuable and allowed clear communication \\
communication & between healthcare professionals \\
Results were reviewed by a second professional, giving rigour to findings and preventing missed & diagnoses \\
Interprofessional trust & There was enhanced interprofessional collaboration and learning between optometrists, \\
ophthalmologists and endocrinologists & Clear protocols that ophthalmologists and the clinic have developed were valuable and \\
& mitigated uncertainty around scope of practice \\
& Being co-located and having regular personal contact between professions assisted building \\
& trust in each other's competency \\
& Two-way referrals - low-risk patients referred from ophthalmologists to the clinic and from \\
& optometrists to ophthalmologist \\
& Willingness to engage with other hospital clinicians-optometrists were accommodating of \\
& inpatient or urgent cases \\
Benefits of shared care & Participants had positive regard for the optometrists \\
for eye professionals & Optometrists experienced professional development \\
\hline
\end{tabular}

\section{Other themes}

$\begin{array}{ll}\text { Unexpected benefits } & \text { Greater access for patients with a range of other complex vision related conditions } \\ \text { Challenges } & \text { Insufficient referrals } \\ & \text { Lower than expected number of patients referred from hospital endocrinologists } \\ & \text { Fospilure to elicit many referrals from local GPs } \\ & \text { Perceptions of demand for services }\end{array}$

GPs, general practitioners.

involvement with the clinic. Interviews lasted between 15 and $30 \mathrm{~min}$.

All nine of the invited clinic staff employed by the CFEH participated in two focus groups $(n=4$ and $n=5)$. Participants were all experienced optometrists (females $n=6$ ). Focus groups lasted for an hour each. Optometrists in the focus groups were all closely involved in the development of the model of care and/or had worked in the clinic.

Ten community-based optometrists took part in the phone survey out of the 29 practices contacted in the local district. No two optometrists were from the same practice. We analysed the data, using the framework developed from the literature. Results are summarised in table 1 . We also identified unexpected benefits of the initiative and ongoing challenges.

\section{Rationale and drivers for the CFEH clinic at the hospital}

The CFEH optometrists described the rationale and thinking behind the shared care model starting with the overarching purpose of the CFEH:

The goal of the Centre for Eye Health is ... to identify people that are at risk of vision loss and catching it [early] so we can manage it and treat it before they lose vision in the first place. (Participant 6)

The first CFEH clinic based at University of NSW sees patients with a wide range of ophthalmic conditions 
referred primarily from the greater Sydney area and across NSW and the Australian Capital Territory. Focus group participants and ophthalmologists from the hospital explained the history and rationale of the new clinic. The choice of the hospital to be the home of this second CFEH clinic was partly opportunistic and arose from a working relationship with ophthalmologists based in this district. The hospital had a modest eye service prior to the CFEH clinic, limited to mostly patients requiring cataract surgery and referrals from within the hospital. Other eye conditions were mostly referred to other hospitals in the Local Health District with larger, better equipped ophthalmic services. Two interdependent aspects of this lack of capacity were: lack of specialised ophthalmic equipment required for assessment, diagnosis, or treatment of different eye conditions and lack of an ophthalmic trained workforce, as without the specialised ophthalmic equipment, the hospital could not host the ophthalmic registrar training programme run by the Royal Australian and New Zealand College of Ophthalmologists. Provision of the CFEH-funded equipment and optometrists was seen as a clear win for the hosting hospital:

[Hospital] Ophthalmology was able to maintain its registrar training status as a result of us [CFEH] being there, otherwise they would have lost that. I mean, that's something. (Participant 5)

At the same time, endocrinologists from the hospital were concerned that a subset of their patients with diabetes were not having routine eye checks exposing them to a high risk of vision loss. These clinicians approached the hospital ophthalmologists for greater access to on-site consultation for these patients. Vision loss from diabetic retinopathy is a high priority for CFEH as early intervention is known to save sight. Funding by CFEH to supply equipment and trained optometrists to enter into a shared model of care alongside the hospital Eye clinic was seen as serving the aims of both the hospital and $\mathrm{CFEH}$.

\section{Patient-related factors}

The purpose of the CFEH clinic was consistently well articulated by participants stating the clinic's intention was to provide a service for people with diabetes who had no regular eye professional doing their routine check-ups and who were at risk of vision loss from diabetic retinopathy. Patients were described as 'people who fall through the cracks' or 'who do not have a relationship with a community optometrist,' or are 'vague about their checks'.

I would like you to acknowledge the fact that there are quite a few patients [with diabetes] who have not had such care provided [from an established relationship with an optometrist or ophthalmologist], who are reluctant to do so and for whom an immediate referral and immediate follow-up [from the CFEH clinic] ... you know, that's made such care possible. (Participant 12)

The importance of the CFEH clinic in terms of distance and ease of access was mentioned by all participants. The position of the clinic was an important enabler for local patients for whom travel was seen as a burden or undesirable:

[The area] is quite cut off and people kind of stay in that area; they don't like to travel (Participant 1)

The University of NSW clinic is $30 \mathrm{~km}$ from the hospital and takes around $90 \mathrm{~min}$ to reach on public transport. The hospital itself is well serviced by public transport for people living in the local region. Existing clients of the CFEH clinic at the University of NSW who lived near the hospital had given feedback to participants that the easier access was welcome:

Patients definitely comment saying it's really great that I can come here [CFEH clinic] instead of all the way out to University of NSW for the same test, you know, especially locals (Participant 1 )

Referrers based in the hospital's district also appreciated that their patients could be seen locally:

So, from our local optometrists referring to the $\mathrm{CFEH}$ model ... at University of NSW, so from them specifically, they love the fact that the patients can stay local so that itself has been good feedback from the local optometry referrers. (Participant 2)

Greater access to eye services was also discussed at length by the hospital staff regarding the ease of access to the service now available within the hospital. This relates both to facilitating access and timeliness of access.

So, [previously] we dictated letters to GPs ... and we advised the patients [recommending routine eye checks]-more or less relying on the good will of the GP to make sure the patient has such an assessment and relying on the patient to find an optometrist ... Having such a service here has made a lot of difference to patients who often have some disability. Because by the time they have retinopathy, they often have microvascular disease, some cognitive impairment, et cetera, so that's a very good complementary service to other services that we provide... [Patients] need to come to our [endocrinology] clinic and the other clinic $[\mathrm{CFEH}]$ is practically within a metre of this, that's made such care possible. (Participant 12)

As well as outpatients, admitted hospital patients with diabetes have benefited from the accessibility of the CFEH eye service at the hospital.

And it also helped with our inpatients... We have quite a few inpatients who come in ... with highly neglected diabetes or some patients who basically don't look after themselves and for them, having such a clinic in our hospital is a chance to get an ophthalmological 
point of view, otherwise they are highly reluctant to go somewhere else. (Participant 12)

\section{Integrated care model}

Endocrinologists commented on the enhanced written communication that the CFEH had brought for them, comparing it to the often poor or absent communication from community-based optometrists. They valued being notified of when checks are done and being informed of any findings or treatment initiated:

I found it very helpful because the clinic provides us with very comprehensive reports, which are very rare to get from an outside service. So, for example, from the optometrists in the shopping centre, they may write possibly, if we're lucky, some kind of tick the box form or a brief comment. (Participant 12)

I don't get a report from outside [community-based] optometrists so I'm trusting [the findings are] OK. (Participant 14)

Optometrists in the focus groups explained the system of enhanced communication they had for GPs who referred patients to the CFEH clinic:

We have our systems in place so we can communicate with the GPs through their electronic media which most optometrists in practice can't. So, we can send our reports through HealthLink which is e-communication, so that they don't actually get a physical piece of paper, it pops up into their patient management system, so there's an added bonus, I suppose, as to our model is easier for them. (Participant 2)

The advantage of shared information was spoken of at length by the optometrists and ophthalmologists working in the eye service. The scans and test results performed by the optometrists are all accessible electronically to the ophthalmologists so even if they are not physically at the hospital, they can review the findings.

The advantage we've got is they can still dial in and look in the system so if you've got something that's urgent they can dial straight in and look at the images and say, "Yes, I need to see that today or send them off to [city] Hospital or I'll be there in an hour," or whatever needs to be done. (Participant 2)

All results of scans and other tests are reviewed by a second professional, an optometrist or ophthalmologist depending on whether the findings are routine or complex. This was described as giving rigour to the findings and preventing any missed diagnoses.

\section{Interprofessional collaboration and trust}

Participants commented on the enhanced interprofessional collaboration and learning that took place between optometrists, ophthalmologists and endocrinologists as a result of the CFEH clinic, a known benefit of integrated care. Optometrists in one of the focus groups spoke about their own professional development, working in the CFEH clinic at the hospital:

I think the standard of care within the centre staff is ... higher than your general community optometrist, so although scope of practice is much the same, we can all dilate, we can all check for diabetic retinopathy and stage and categorise accordingly... We have direct access to ophthalmological opinion. I get constant feedback on all my cases in writing. I think that makes you grow as a clinician, keeps me engaged and keeps the standard high as well. (Participant 5)

Several participants discussed the value of the clear protocols that the ophthalmologists and CFEH had developed so that such uncertainty could be minimised:

There are very clear guidelines for care, patients can move freely between CFEH and [the hospital] eye clinic and we can adjust that movement. The information is all there to assist decisions. This is definitely not the case when dealing with community-based health providers. (Participant 13)

Participating optometrists and ophthalmologists working in the clinics were asked directly if there had been any issues concerning triaging of patients into the clinics, scope of practice or ongoing management recommendations, all factors that can be negatively affected by a lack of interprofessional trust. Responses were typically positive and included referral of low risk patients from the ophthalmologists to the optometrist in the CFEH clinic:

Interviewer: You are working with optometrists... any issues with that? Participant: More generally [outside of the hospital service] ... There are occasions where you see patients and think they probably should have been referred earlier [by the optometrists], but more often than not, it's the other way around, that patients are referred urgently, they probably don't need to be so urgently referred. With the hospital service, ... that works really well, because we're in the clinic together. So, if there's any questions, we're just around the corner and it's easy to ask. So, you don't get delayed diagnoses and things like that, because if it's a little bit iffy, they'll just get us to have a look and that works really well that way. (Participant 13)

Participants commented on how interprofessional trust in the CFEH clinic setting was increased by a willingness to engage with the other health professionals:

The optometrists are very accommodating of inpatient or urgent cases. They will squeeze them in if they can (Participant 14)

If I've got questions regarding a finding it helps to start with the optometrist or ophthalmology registrar, it makes so much difference that you can clarify the issue when the patient is there and getting immediate advice. (Participant 12) 
Interprofessional trust was also increased by the positive regard that participants had for the optometrists:

I would like to say that I've always noticed how really professional and lovely Centre for Eye Health staff are... They're a good bunch of people to work with. (Participant 10)

\section{System and service-level factors}

Some participants commented on how the shared care arrangement and close proximity of the two professions allowed more efficient operation of the eye service as a whole:

Using ophthalmologists to screen low risk patients is not efficient and has an impact on the hospital so it makes sense to use optometrists. It also helps alleviate the pressure the hospital is under to keep waitlists down for the eye clinic. (Participant 13)

\section{Unexpected benefits}

An unexpected benefit of the establishment of the CFEH clinic at the hospital and their provision of specialised ophthalmic assessment equipment was the greater access for patients with a range of other complex vision-related conditions.

We're seeing a fair few neurology referrals in the clinic. And that's, I think, mainly because there's been a field machine become available and so, they want to know about the fields and are asking us to see those patients, which previously they wouldn't have asked us to see. (Participant 13)

[The eye service] also provides assessments for more complicated patients, endocrine patients with pituitary tumours. ... We've already had quite a few patients who in order to get such assessments previously had to go [travel $30 \mathrm{~km}$ ] to [city] Hospital and not only were patients unwell, they may have had a disability and we had to ... wait sometimes for several months ... [But] a phone call, right here, everything is being done smoothly and allows us to provide safer and more comprehensive care. (Participant 12)

\section{Challenges}

The main risk to the continuing operation of the CFEH clinic at the hospital was insufficient referrals:

Not getting enough patients through the clinic, I suppose, is the main risk. (Participant 13)

Other problems concerned hospital-controlled resources. For example, governance processes needed for formal approvals to use fluorescein angiography and the retinal laser in the clinic had not been actioned by the hospital. Employment of an additional staff member in the hospital Eye clinic, paid for by CFEH had also been delayed pending hospital approvals.

Issues around perceptions of demand for services seemed to be a contributing factor. Hospital staff noted
Table 2 Demographics of survey participants

\begin{tabular}{lll}
\hline Variable & N & \% \\
\hline Gender & & \\
Male & 4 & 44 \\
Female & 5 & 55 \\
Not specified & 1 & 10 \\
\hline Age & & \\
\hline 20s & 6 & 60 \\
30s & 2 & 20 \\
40s & 2 & 20 \\
Experience (years) & & \\
\hline 3-5 & 6 & 60 \\
$6-10$ & 2 & 20 \\
$>10$ & 2 & 20 \\
\hline
\end{tabular}

concerns by hospital management that clinic resources would be stretched by the diabetic retinopathy service. By contrast, the hospital staff believed that the collaborative arrangement with CFEH maximised the use of hospital resources by diverting low-risk patients to the CFEH clinic and that pressure on hospital resources arose from other sources.

\section{Survey}

Ten community-based optometrists took part in the phone survey. Participants' demographics are presented in table 2 . Respondents were equally split between male and female. Most had been working in optometry for 3-5 years $(\mathrm{n}=6,60 \%)$, two for $<10$ years and two over 10 years. All except one indicated they had heard of the CFEH clinic at the hospital. Demographics of their practice were predominantly 'Caucasian' or 'European' with one practice including 10\% Middle Eastern. All respondents stated that fewer than $1 \%$ of their clients were from an Aboriginal or Torres Strait Islander background, one noting these clients 'mostly use the Aboriginal Medical Service'. Most common conditions seen were cataracts, and macular degeneration.

Most respondents planned to refer to the clinic at the hospital $(n=8,80 \%)$ with the remainder not sure. The reasons given for not referring were (quoting written notes by survey facilitator):

Not sure of the process; the ophthalmologist will cover more bases and patient will gain a greater advantage by seeing the specialist rather than opinion [of the optometrist] (Optom\#5)

If there was treatment [for diabetic retinopathy] available at the CFEH clinic, would be more likely to refer to them. The clinic doesn't offer anything our practice does not offer (Optom\#1)

Half the respondents $(n=5)$ would consider referring clients with diabetic retinopathy to the CFEH clinic at the hospital. Three said 'no' and two were unsure. When 


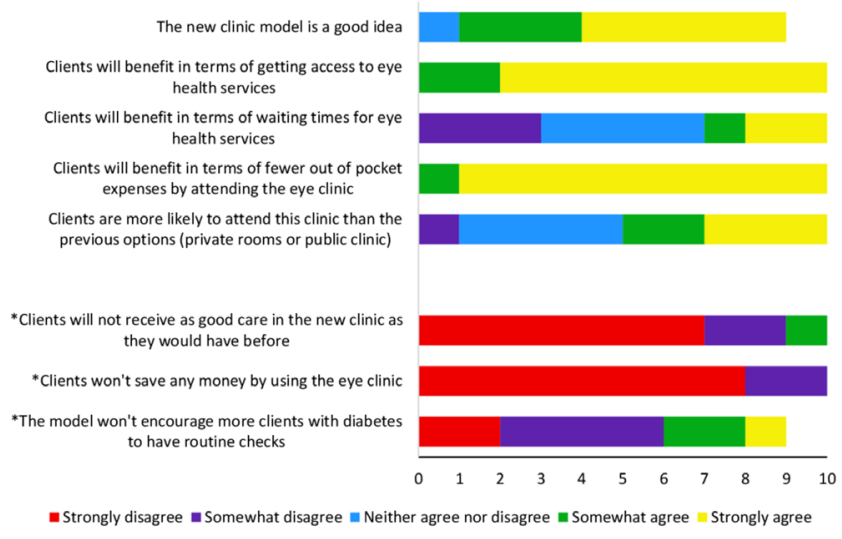

Figure 1 Responses to statements about the new CFEH clinic at the hospital. *Statements are worded negatively. CFEH, Centre for Eye Health.

asked what clients they thought were best suited to the clinic, five replies given all focused on cost: those who cannot afford a specialist. All respondents stated they knew how to refer to the clinic.

Respondents were asked to consider eight statements about the clinic on a 5-point Likert scale from strongly disagree to strongly agree (figure 1 ). The strongest agreement was for the statements: 'Clients will benefit in terms of waiting times for eye health services' and 'Clients will benefit in terms of fewer out-of-pocket expenses by attending the eye clinic'. The strongest disagreement was for the statement, phrased as the reverse of an earlier one about cost: 'Clients won't save any money by using the eye clinic'. Opinions were divided over the statement: 'The model won't encourage more clients with diabetes to have routine checks'.

\section{DISCUSSION}

We have shown that the CFEH clinic at the hospital addressed barriers known to affect assessment rates for people with diabetic retinopathy, including improved access (both geographic, ${ }^{21} 41$ and ease for people with disability), assistance for patients unable or unwilling to organise their own eye checks, and efficient management of human resources. ${ }^{22} 3042$ The clinic has also optimised several of the drivers of this model of care, providing a clear scope of practice and protocols for shared care between optometrists and ophthalmologists, ${ }^{34}$ good communication between referrers and eye professionals, ${ }^{29}$ and a collegial approach that is promoting interprofessional trust. $^{22}$ Areas of concern are the lack of referrals from GPs, lower than expected numbers of referrals from the hospital endocrinologists and some issues with stretched administrative capacity. There was also qualified discussion around some perceived mismatches between the concerns of hospital management regarding pressure on resources on the one hand, and the aims of the clinic on the other. Local optometrists were largely supportive of the aims of the clinic, stating they believed clients would benefit. Only three of our sample of ten stated they would not consider referring their own clients to the clinic.

The published literature provides ample evidence that access to appropriate, timely and acceptable eye services is a major facilitator for regular assessment for diabetic retinopathy. ${ }^{7}$ Integrated care models seek to solve barriers such as difficult access ${ }^{21} 22$ or mitigate patientlevel issues such as lack of education about diabetic eye disease or a lack of capacity to organise their own check-ups. ${ }^{18-20} 4243$ Integrated care is an acknowledged facilitator of assessment for diabetic retinopathy, overcoming problems associated with poor communication, fragmented services, unshared information and extra burden for the patients. ${ }^{22} 24-29$ All participants discussed the comprehensive nature of the report that is returned to the referrers and agreed on its value in allowing clear communication between all health professionals sharing care of patients.

A barrier to shared models of care that is frequently reported in the literature is lack of interprofessional trust between optometrists and ophthalmologists. ${ }^{22} 30$ One aspect of this concerns uncertainty around scope of practice: who is responsible for what, and when care should be transferred. The CFEH had mitigated this barrier by co-developing robust protocols that defined these things clearly. This removed uncertainty and allowed clearly understood processes.

Interprofessional trust is a key issue with integrated care models. The model used at the Royal Victorian Eye and Ear Hospital experienced some issues in this regard as ophthalmologists had no way of assessing the competency of community-based optometry partners except by frequent testing which the optometrists found somewhat patronising and inefficient. ${ }^{31}$ The CFEH clinic, being co-located and having regular personal contact between professions, seems to have mitigated this problem. There was ample evidence of respect for each other's competencies.

Lower than expected referrals were discussed by participants as primarily due to the lower than expected number of patients being referred by the hospital endocrinologists, and failure to elicit many referrals from local GPs. The risk of insufficient referrals was being addressed through greater engagement with GPs. Optometrists in their focus group provided evidence of strategies tried, including visits to individual practices, newsletters and phone calls, and crafting of integrated e-communication. In addition, all 53 local area GP practices were contacted by phone during the study, and were asked if their GPs were interested in taking part in the phone survey. We were unable to recruit any participants, hence our focus on optometry and endocrinology referrals.

This model of care came about through the unique situation of an outer metropolitan hospital with limited eye services, and local patients seen to be at high risk of diabetic retinopathy. Supported by hospital endocrinologists who saw some of their vulnerable patients 'slipping through the cracks' CFEH saw how equipping and 
staffing a clinic at the hospital was firmly aligned with their primary goal of preventing vision loss. While such shared models of eye care are not unknown in Australia, it is rare to find optometrists working at a hospital, co-located with ophthalmologists, and we would argue that the close relationships between endocrinologists and eye clinic staff are also unique.

\section{Limitations and strengths}

We invited as many stakeholders in the clinic as we could identify and had an excellent response from them (only two of the hospital staff did not respond). All participants were familiar with the clinic and had been working within the model of care for some time. As such, they were excellent informants able to give first-hand observations of how it was working from their individual viewpoint. Many participants were also able to report feedback from patients and referrers. This made them valuable participants. On the other hand, it could be argued that the sample of participants was biased in favour of the clinic, as all were either instrumental in the clinic's set up or are actively engaged with it. The flagging of areas of nonengagement (eg, from local GPs) and difficulties related to limited hospital-controlled resources are potential future areas to explore with interviews or focus groups. Data were collected from a unique context and model of care, and as such may not be generalisable. Value of the data lies in its potential to inform future partnerships of optometrists with other organisations.

Despite a variety of approaches, we failed to recruit any GPs to participate in the telephone survey. After unsuccessful attempts to engage participants via the telephone, we advertised the survey on the CFEH newsletter received by all the general practices, and allowed access to the survey via a $\mathrm{QR}$ code. However, no responses were recorded. We note other research groups that have had difficulty recruiting $\mathrm{GPs}^{44}$ and acknowledge that their role as referrers to all specialist services makes participation in the large number of research requests they received difficult. Our inability to collect data from the GPs in the area means we have no first-hand information about the acceptability of the clinic from their perspective, and in this phase of the work we do not report on the perceptions of patients.

\section{CONCLUSION}

The new model was considered to have addressed many of the barriers to assessment such as providing local easy access to services, integrated care with an interdisciplinary team, with clear scopes of practice and guidelines, and shared communication. While there remain issues with the model, there were also unexpected benefits, such as providing an additional diagnostic service to inpatients, and ad hoc consultations for patients with eye problems attending the endocrinology clinic.

Twitter Janet C Long @JanetCLong, Zeyad Mahmoud @zeyadmahmoud and Robyn Clay-Williams @RClaywilliams
Acknowledgements We thank Sean Siveng and Joanna Taylor for assistance with the data collection. We also thank all our participants.

Contributors JCL, BB, RC-W and JB conceived and designed the project. JCL, BB and ZM drafted the paper and undertook data collection and analysis. MK, BZ and $\mathrm{AL}$ contributed to design and analysis, and critically reviewed the manuscript. MY and NA critically reviewed the manuscript. All authors read and approved the final manuscript.

Funding This work was supported by CFEH.

Competing interests JCL, BB and ZM were supported by a grant from the CFEH to undertake the evaluation. MK, AL, BZ and MY have joint appointments with the CFEH and University of NSW.

Patient consent for publication Not required.

Ethics approval Ethical and research governance approval was obtained from the South Eastern Sydney Local Health District (SESLHD) HREC ref 17/333 (LNE/18/ POWH/14).

Provenance and peer review Not commissioned; externally peer reviewed.

Data availability statement No data are available. Focus group and interview schedules are provided but full transcripts are not due to Ethics stipulations around confidentiality of participants.

Open access This is an open access article distributed in accordance with the Creative Commons Attribution Non Commercial (CC BY-NC 4.0) license, which permits others to distribute, remix, adapt, build upon this work non-commercially, and license their derivative works on different terms, provided the original work is properly cited, appropriate credit is given, any changes made indicated, and the use is non-commercial. See: http://creativecommons.org/licenses/by-nc/4.0/.

ORCID iDs

Janet C Long http://orcid.org/0000-0002-0553-682X

Zeyad Mahmoud http://orcid.org/0000-0003-3331-8093

Robyn Clay-Williams http://orcid.org/0000-0002-6107-7445

Jeffrey Braithwaite http://orcid.org/0000-0003-0296-4957

\section{REFERENCES}

1 Australian Bureau of Statistics. National health survey, 2019.

2 Australian Institute of Health Welfare. Older Australia at a glance, 2017.

3 Commonwealth of Australia. 2015 intergenerational report: Australia in 2055: Commonwealth of Australia Canberra, 2015.

4 McPhail SM. Multimorbidity in chronic disease: impact on health care resources and costs. Risk Manag Healthc Policy 2016;9:143-56.

5 Vijan S, Hayward RA, Ronis DL, et al. Brief report: the burden of diabetes therapy. J Gen Intern Med 2005;20:479-82.

6 Taylor HR, Pezzullo ML, Keeffe JE. The economic impact and cost of visual impairment in Australia. British Journal of Ophthalmology 2006;90:272-5.

7 Liew G, Michaelides M, Bunce C. A comparison of the causes of blindness certifications in England and Wales in working age adults (16-64 years), 1999-2000 with 2009-2010. BMJ Open 2014;4:e004015.

8 Mitchell P, Foran S, Wong TY, et al. Guidelines for the management of diabetic retinopathy. Canberra, Australia: National Health and Medical Council, 2008.

9 Turner AW, Mulholland WJ, Taylor HR. Coordination of outreach eye services in remote Australia. Clin Experiment Ophthalmol 2011;39:344-9.

10 Victorian Agency for Health Information. Statewide - Median wait time for routine specialist clinic appointments - Ophthalmology Quarterly Data: Victorian State Government, 2019. Available: https:// performance.health.vic.gov.au/Home/Report.aspx?ReportKey=677 [Accessed 2 Sep 2019].

11 Centre for Eye Health. About CFEH 2019. Available: https://www. centreforeyehealth.com.au/about/ [Accessed 25 June 2019].

12 Kodner DL, Spreeuwenberg C. Integrated care: meaning, logic, applications, and implications - a discussion paper. Int J Integr Care 2002;2:e12.

13 Evans JR, Michelessi M, Virgili G. Laser photocoagulation for proliferative diabetic retinopathy. Cochrane Database of Systematic Reviews 2014;11.

14 Nguyen QD, Brown DM, Marcus DM, et al. Ranibizumab for diabetic macular edema: results from 2 phase III randomized trials: rise and ride. Ophthalmology 2012;119:789-801. 
15 Korobelnik J-F, Do DV, Schmidt-Erfurth U, et al. Intravitreal aflibercept for diabetic macular edema. Ophthalmology 2014;121:2247-54.

16 Larizza MF, Hodgson LA, Fenwick EK, et al. Feasibility of screening for diabetic retinopathy at an Australian pathology collection service: a pilot study. Med J Aust 2013;198:97-9.

17 Foreman J, Keel S, Xie J, et al. Adherence to diabetic eye examination guidelines in Australia: the National eye health survey. Med J Aust 2017;206:402-6.

18 Lewis K, Patel D, Yorston D, et al. A qualitative study in the United Kingdom of factors influencing attendance by patients with diabetes at ophthalmic outpatient clinics. Ophthalmic Epidemiol 2007;14:375-80.

19 Hartnett ME, Key IJ, Loyacano NM, et al. Perceived barriers to diabetic eye care: qualitative study of patients and physicians. Archives of Ophthalmology 2005;123:387-91.

20 Hipwell AE, Sturt J, Lindenmeyer A, et al. Attitudes, access and anguish: a qualitative interview study of staff and patients' experiences of diabetic retinopathy screening. BMJ Open 2014;4:e005498.

21 Lee DJ, Kumar N, Feuer WJ, et al. Dilated eye examination screening guideline compliance among patients with diabetes without a diabetic retinopathy diagnosis: the role of geographic access. BMJ Open Diab Res Care 2014:2:e00031.

22 Lindenmeyer A, Sturt JA, Hipwell A, et al. Influence of primary care practices on patients' uptake of diabetic retinopathy screening: a qualitative case study. British Journal of General Practice 2014;64:e484-92.

23 Graham-Rowe E, Lorencatto F, Lawrenson JG, et al. Barriers to and enablers of diabetic retinopathy screening attendance: a systematic review of published and grey literature. Diabetic Medicine 2018;35:1308-19.

24 Griffin-Shirley N, Trusty S, Kelley P, et al. Barriers to eye care faced by adult Hispanics with diabetes. RE:view: Rehabilitation and Education for Blindness and Visual Impairment 2004;36:53-62.

25 Kizor-Akaraiwe NN, Ezegwui IR, Oguego N, et al. Prevalence, awareness and determinants of diabetic retinopathy in a screening centre in Nigeria. J Community Health 2016;41:767-71.

26 Moss SE, Klein R, Klein BE. Factors associated with having eye examinations in persons with diabetes. Arch Fam Med 1995;4:529-34.

27 van Eijk KND, Blom JW, Gussekloo J, et al. Diabetic retinopathy screening in patients with diabetes mellitus in primary care: incentives and barriers to screening attendance. Diabetes Res Clin Pract 2012;96:10-16.

28 Yuan Z. Risk factors and barriers to eye care services among presenting late diabetic retinopathy patients in Shanxi Province in China, 2007.

29 Kashim RM, Newton P, Ojo O. Diabetic retinopathy screening: a systematic review on patients' Non-Attendance. Int J Environ Res Public Health 2018;15
30 Shah M, Noor A, Deverell L, et al. Task sharing in the eye care workforce: screening, detection, and management of diabetic retinopathy in Pakistan. A case study. Int $J$ Health Plann Manage 2018;33:627-36.

31 O'Connor PM, Harper CA, Brunton CL, et al. Shared care for chronic eye diseases: perspectives of ophthalmologists, optometrists and patients. Medical Journal of Australia 2012;196:646-50.

32 White A, Goldberg I, Australian and New Zealand Glaucoma Interest Group and the Royal Australian and New Zealand College of Ophthalmologists. Guidelines for the collaborative care of glaucoma patients and suspects by ophthalmologists and optometrists in Australia. Clin Exp Ophthalmol 2014;42:107-17.

33 Shah M, Noor A, Ormsby GM, et al. Attitudes and perceptions of eye care workers and health administrators regarding task sharing in screening and detection for management of diabetic retinopathy in Pakistan. Ophthalmic Epidemiol 2018;25:169-75.

34 Shah M, Noor A, Ormsby GM, et al. Task sharing: development of evidence-based co-management strategy model for screening, detection, and management of diabetic retinopathy. International Journal of Health Planning and Management 2018.

35 National Health and Medical Research Council. NHMRC guidelines for the screening, prognosis, diagnosis, management and prevention of glaucoma, 2010.

36 Optometry Board of Australia. Guidelines for use of scheduled medicines, 2018. Available: https://www.optometryboard.gov.au/ Registration-Standards/Endorsement-for-scheduled-medicines. aspx

37 Lu CY, Lu VH, Goldberg I, et al. Towards integrated care: Australia's new model of care for patients with glaucoma. Med J Aust 2010;193:200-1.

38 Gray SF, Spencer IC, Spry PG, et al. The Bristol shared care glaucoma study - validity of measurements and patient satisfaction. $J$ Public Health 1997;19:431-6.

39 Bhargava JS, Bhan-Bhargava A, Foss AJE, et al. Views of glaucoma patients on provision of follow-up care; an assessment of patient preferences by conjoint analysis. Br J Ophthalmol 2008;92:1601-5.

40 Banes MJet al. Agreement between optometrists and ophthalmologists on clinical management decisions for patients with glaucoma. British Journal of Ophthalmology 2006;90:579-85.

41 Lindenmeyer A, Sturt JA, Hipwell A, et al. Influence of primary care practices on patients' uptake of diabetic retinopathy screening: a qualitative case study. Br J Gen Pract 2014;64:e484-92.

42 Sachdeva A, Stratton IM, Unwin J, et al. Diabetic retinopathy screening: study to determine risk factors for non-attendance. Diabetes \& Primary Care 2012;14.

43 Roy MS. Eye care in African Americans with type 1 diabetes: the new Jersey 725. Ophthalmology 2004;111:914-20.

44 Williamson MK, Pirkis J, Pfaff JJ, et al. Recruiting and retaining GPs and patients in intervention studies: the DEPS-GP project as a case study. BMC Med Res Methodol 2007;7:42. 